\title{
Migration of Silicone Gel into Breast Parenchyma Following Mammary Prosthesis Rupture
}

Louis C. Argenta, M.D.

Ann Arbor, Michigan

\begin{abstract}
The case report of a patient with a ruptured gel mammary prosthesis complicated by migration of the gel into the breast parenchyma is presented. It is felt that the constant massage of the prostheses in an attempt to avoid fibrous contracture after closed capsulotomy is the etiology for this migration. The prosthesis and extruded gel were removed by sacrificing breast parenchyma. Repeated prosthesis massage after closed capsulotomy exposes the patient to significant potential complication if the prosthesis has been ruptured.
\end{abstract}

Key words: Prosthesis rupture - Silicone migration

Despite a long list of potential complications, augmentation mammoplasty continues to be a greatly demanded and frequently performed procedure. Fibrous capsular contracture with firmness and distortion of the breast remains the most frequent complication. Many techniques have been developed in an attempt to treat these patients. The most widely used procedure is closed capsulotomy [1] and prosthesis massage exercises [4].

Complications from closed capsulotomy include hematoma, incomplete rupture of the capsule resulting in abnormal shapes of the breast, and rupture of the prostheses $[2,3]$. We report here another complication-the rupture of an implant with subsequent migration of get into the breast parenchyma. Repeated prosthetic massage following closed capsulotomy are felt to be the factors responsible for this complication.

Address reprint requests to Louis C. Argenta, M.D., Assistant Professor of Surgery. Section of Plastic Surgery, University of Michigan Hospital, Ann Arbor, Michigan 48109. USA

\section{Case Report}

A 26-year-old woman underwent augmentation mammoplasty for postpartum atrophy of the breasts in April 1975. Heyer-Schulte 180-cc gel prostheses were placed in the retromammary space. No steroids or other medications were used. The patient did extremely well with a minimal amount of bilateral firmness until 1980. At that time bilateral Baker III capsules were treated with closed capsulotomy successfully. This was followed by a series of massage exercises in which the patient was encouraged to roll on a carpeted floor putting the weight of her thorax on the prostheses. Minor recurrences of firmness were treated with closed capsulotomies by the patient's husband on approximately 3 occasions. The patient enthusiastically pursued the exercises noting that both breasts had become significantly softer. Nine months after the initial capsulotomy, the patient noticed firmness in the superior aspect of the right breast. Examination raised the suspicion of possible neoplasm, and mammograms were performed. Mammograms in 2 projections revealed rupture of the prostheses with a large number of nodules of silicone within the breast tissue.

In November 1981, an attempt was made at extracapsular excision of the ruptured prostheses. Multiple areas where the capsular membrane had been ruptured were encountered and poorly encapsulated gel was removed by sacrificing surrounding breast tissue. No prosthesis was inserted since it was impossible to determine if all extravasated gel had been removed. Two months after this procedure the breast was clinically soft and without evidence of masses. Mammograms failed to demonstrate any residual gel within the breast. The patient was returned to the operating room where a doublelumen $220 \mathrm{-cc}$ prosthesis was placed into the retromammary space to give symmetry with the opposite 


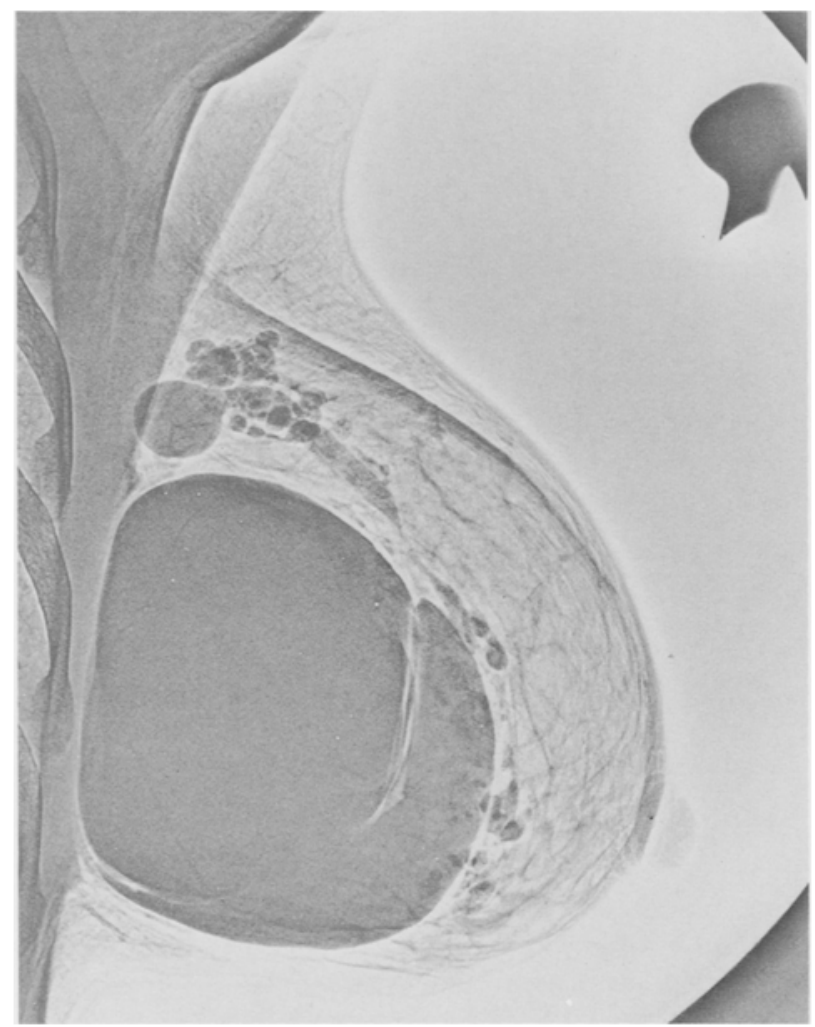

Fig. 1. Lateral mammogram demonstrating convolutions in the prosthesis secondary to rupture of the prosthesis. Multiple nodules of radio-dense material are observed within the parenchyma of the breast

breast. The patient tolerated the procedure well and has had an uncomplicated postoperative course.

\section{Discussion}

The incidence of prosthesis rupture following closed capsulotomy is unknown, but is generally felt to be less than $1 \%$. With an inflatable prosthesis, the sequelae of the rupture is obvious with total deflation within a period of several days. One of the postulated benefits of the gel prosthesis has been that if rupture occurs, the cohesiveness of the gel and fibrous capsule will contain the extruded gel. If, however, secondary closed capsulotomies or prosthesis massage follow rupture, the extruded gel may be propelled through the capsulotomy rent and into the breast parenchyma. The development of a soft breast may, as in our case, encourage the patient to pursue vigorously an exercise regimen causing further migration of the gel.

While mammograms after every closed capsulot-

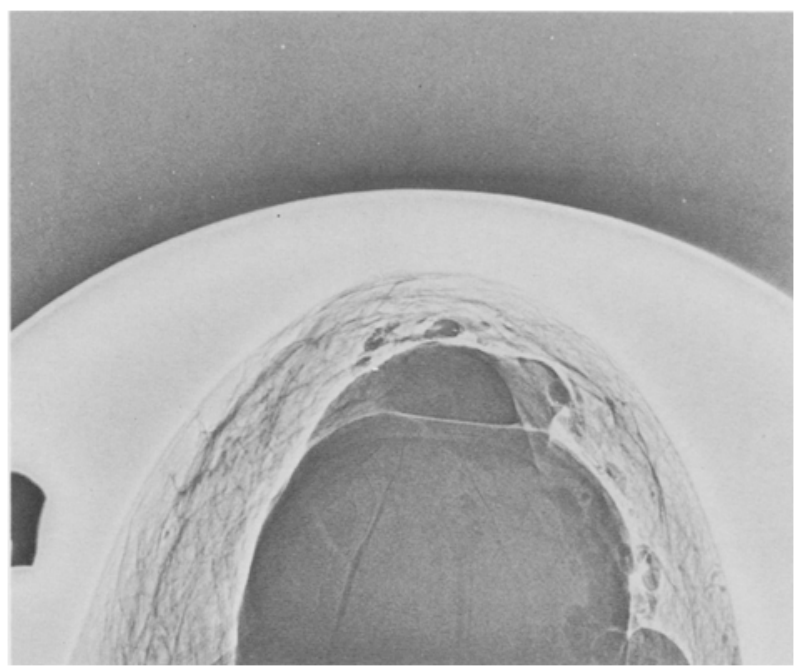

Fig. 2. Superior-inferior projection mammogram demonstrating multiple convolutions in the prostheses confirming the diagnosis of a ruptured prosthesis. Again, multiple nodules of silicone are seen within the breast parenchyma

omy are obviously excessive. development of nodularity or breast abnormality in a patient who has undergone augmentation mammoplasty and closed capsulotomy warrants workup. Mammograms in 2 projections may provide insights into the integrity of the prosthesis.

It has been our feeling that ruptured prostheses should be removed expeditiously. We have removed several such prostheses without excessive difficulty by extracapsular dissection. In most situations, the gel is well contained and the prostheses can be reinserted at the same operation. Migration of the gel out of the pseudo-capsule makes removal of the material much more difficult and requires the removal of breast parenchyma.

\section{References}

1. Baker JL, Bartels RJ, Douglas WM: Closed compression technique for rupturing a contracted capsule around a breast implant. Plast Reconstr Surg 58:137. 1976

2. Eisenberg HV, Bartels RJ: Rupture of a silicone baggel breast implant by closed compression capsulotomy. Plast Reconstr Surg 59:849, 1977

3. Feliberti MC, Arrillaga A, Colon GA: Rupture of inflated breast implants in closed compression capsulotomy. Plast Reconstr Surg 59:848, 1977

4. Vinnik CA: Spherical contracture of fibrous capsules around breast implants. Plast Reconstr Surg 58:555. 1976 